\title{
COMPARISON OF DIFFERENT CLUSTERING ALGORITHMS VIA GENETIC ALGORITHM FOR VRPTW
}

\author{
Gocken, T. ${ }^{\#}$ \& Yaktubay, M. \\ Department of Industrial Engineering, Adana Alparslan Turkes Science and Technology University, \\ Adana, Turkey \\ E-Mail: tgocken@atu.edu.tr ( ${ }^{\sharp}$ Corresponding author)
}

\begin{abstract}
In this paper, Vehicle Routing Problem with Time Windows (VRPTW) with known customer demands, a central depot and a set of vehicles with limited capacity, is considered. The objectives are both to minimize the total distance and the total waiting time of the vehicles while capacity and time windows constraints are secured. The applied solution techniques consist of three steps: clustering, routing and optimizing. By using K-means, Centroid-based heuristic, DBSCAN and SNN clustering algorithms in the initial population generation phase of genetic algorithm, the customers are divided into feasible clusters. Then feasible routes are constructed for each cluster. Lastly, the feasible route solutions are taken as the initial population and genetic algorithm is utilized for the optimization. A set of well-known benchmark data is used to compare the obtained results. According to the results of the study it is observed that using K-means clustering algorithm in generating the initial population of the genetic algorithm is more effective for the handled problem.

(Received in April 2019, accepted in October 2019. This paper was with the authors 1 month for 1 revision.)
\end{abstract}

Key Words: Vehicle Routing with Time Windows, Genetic Algorithm, Clustering, Multi-Objective Optimization, K-means Clustering Algorithm

\section{INTRODUCTION}

Vehicle Routing Problem with Time Windows (VRPTW) which is a type of classical Vehicle Routing Problem (VRP) handles a transportation issue that is comprised in the logistics management which is a substantial component of the supply chain management. VRPTW searches optimum routes for a fleet of vehicles making delivery from a depot to the customers in a specified time interval. Route optimization has a significant importance in logistics management owing to the effect on the customer satisfaction by fast delivery and lower cost. In accordance with this concept, VRPTW is concentrated on minimizing total travelled distance and usage of the vehicles, which are directly related with economic and environmental sustainability.

Many approaches that have utilized exact, heuristic and metaheuristic algorithms have been developed heretofore to solve VRPTW which is a hard-combinatorial problem. If the size of the customer set is small, the exact algorithms can be utilizable; else if the set is getting larger it is not viable to use these algorithms due to the high solution duration. For this reason, the solution approaches of the problem via heuristic and metaheuristic strategies are growing in the literature recently. Genetic Algorithm (GA) is frequently used in the solution of the VRP and VRPTW. It is a useful tool to produce good solutions to optimization and search problems. GA iteratively improves a set of solutions by mimicking the biological evolution in natural selection. Because GA is a versatile and effective approach, it is prone to the hybridization. It aims to increase the probability of getting the best solution of an optimization problem and to decrease the time of searching. In the literature of the VRP and VRPTW, there are plenty of studies that applied GA incorporated with other heuristics [1-7].

In the literature, there are also studies that utilized clustering algorithms in the routing problem. Díaz-Parra et al. [8] used K-means clustering algorithm and neighbourhood techniques to produce a non-random initial population for GA and solved a VRPTW. Comert 
et al. [9] proposed a hierarchical approach for solving VRPTW in a real life situation. At first, the customers are clustered by K-means, K-medoids and DBSCAN clustering algorithms separately. Then, the routes are formed with an exact method. Shin and Han [10] proposed a 3-phase centroid-based heuristic algorithm for VRP. Their algorithm constructs clusters first, then adjusts the clusters, at last establishes the routes. They presented that, their proposed algorithm achieves better results than the sweep algorithm. López-Santana et al. [11] considered a problem of scheduling and routing in a courier service. Before the construction of the routes, the customers are clustered by a centroid-based algorithm and a sweep algorithm. Min et al. [12] proposed a three-stage algorithm for split delivery VRP. First, maximum-minimum distance method is employed to cluster customers. Second, load-demand adjustment is employed. Third, a tabu search algorithm is used to optimise the routes. Zhang [13] used a density based clustering algorithm, i.e. improved DBSCAN, in the solution approach for a VRP. Erdogan and Miller-Hooks [14] proposed a solution approach for a green VRP. They developed a density based clustering algorithm in a part of their approach. It has been built based on DBSCAN algorithm.

In this study, the effect of the different clustering algorithms on the GA performance is analysed by using them at the initial population generation step. K-means, centroid-based heuristic, DBSCAN and SNN clustering algorithms are used at the initial population generation step. According to the best of our knowledge, there is not a comparable study measuring the effect of these partitioning and density based clustering algorithms on GA for solving multi-objective vehicle routing problem with capacity and time windows constraints. The algorithms have been tested on Solomon's well-known benchmarking problems of VRPTW literature.

\section{PROBLEM DEFINITION}

VRP is the problem of forming optimal routes to the vehicles that will serve a customer set. The information of the customers and the $\operatorname{depot}(\mathrm{s})$ (e.g. numbers, demand quantities, geographic data) are known before starting the solution of the problem. The vehicles that serve the customers are assumed to be composed of a homogeneous fleet with a capacity limitation. Each vehicle begins on the route from the depot and returns to the depot at the end of the route. The requirements of each customer must be met in one single vehicle at a time. The total demand of the customers which served by the same vehicle should not be in excess of the capacity limit of the vehicle.

VRP with only one restriction, i.e. vehicle capacity restriction, refers to the Capacitated Vehicle Routing Problem (CVRP). The components which all are found in CVRP, i.e. a homogeneous fleet with specific number of vehicles with the same capacity and characteristics, customers with known demands and locations, a warehouse with a known geographical location, are exist also in VRPTW. The constraint that makes VRPTW different and challenging from other VRP varieties is that there is a specific time interval $\left(e_{i}, l_{i}\right)$ at which service can start for each customer. This time interval is the time window constraint of each relevant customer. The time window of the depot signifies the maximum traveling time of the individual routes. Making delivery or providing services to each customer takes as long as service duration $s_{i}$. At the end of the service duration, the vehicle drives to the next customer or the depot [5].

In this study, minimization of the total distance and minimization of the total waiting time of the vehicles are determined as the objective functions and the problem is modelled as a multi-objective optimization problem.

The mathematical model of the problem is presented in the study of Gocken et al. [5]. 


\section{PROPOSED APPROACH}

The applied solution techniques in our study consist of three steps: clustering, routing and optimizing. The customers are clustered as groups, taking into consideration of the vehicle capacity constraint. To get initial feasible solutions the grouped customers are routed according to the time window constraint. The solutions are optimized via GA beginning with these initial solutions.

By using K-means, Centroid-based heuristic, DBSCAN and SNN clustering algorithms in the initial population generation phase of GA, the customers are divided into feasible clusters. Then feasible routes are constructed for each cluster. Lastly, the feasible route solutions are taken as the initial population and GA is utilized for the optimization.

\subsection{Clustering phase}

(1) K-means

$\mathrm{K}$-means is a partitioning clustering algorithm and developed by MacQueen in [15]. Kmeans algorithm partitions the set of objects into $K$ clusters. The name of the algorithm comes from the parameter $K$, the number of clusters. The steps of K-means algorithm are shown in Table I.

Table I: Steps of K-means algorithm.

\begin{tabular}{|l|}
\hline Parameter: $k$ \\
\hline 1: Select $k$ random points as centroids i.e. initial cluster centres. \\
2: repeat \\
3: Assign each point to the closest centroid's cluster. \\
4: $\quad$ Check the feasibility of capacity constraint. \\
5: Recalculate the centroid of each cluster. \\
6: until Centroids do not change. \\
\hline
\end{tabular}

(2) Centroid-based heuristic

Shin and Han [10] proposed a 3-phase Centroid-based heuristic algorithm to solve the CVRP. The phases are cluster construction, cluster adjustment and route establishment. In this study, cluster construction and cluster adjustment phases are utilized for clustering the customers. The steps of Centroid-based heuristic algorithm are shown in Table II. The steps 1-11 represent cluster construction phase and 12-16 represent cluster adjustment phase.

Table II: Steps of Centroid-based heuristic algorithm.

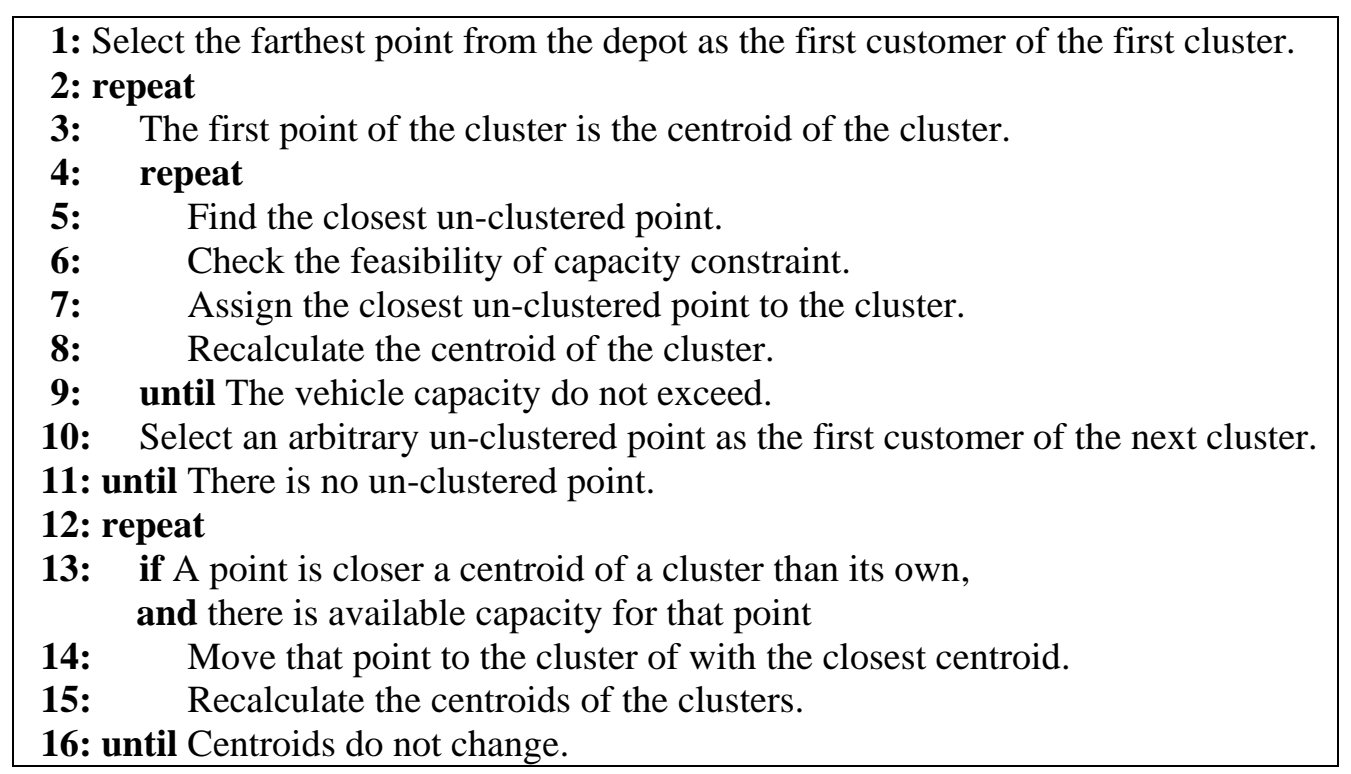




\section{(3) DBSCAN}

Density based clustering algorithm named as Density based Spatial Clustering of Application with Noise (DBSCAN) proposed by Ester et al. [16]. The density of a point is defined as the number of points, that is, neighbours, located within a specified radius (Eps) from that point. The main idea is to form the clusters that have at least MinPts points within the Eps neighbourhood. Eps neighbourhood of a point p, denoted by $\mathrm{N}_{E p s}(\mathrm{p})$, is the class of the points that have at most Eps distance from p. The points which have at least MinPts neighbours in its $\mathrm{N}_{E p s}$ class are defined as core points. The core points are directly densityreachable to their neighbours in $\mathrm{N}_{E p s}$ class. The points that are non-core points but are in the $\mathrm{N}_{E p s}$ class of a core point are defined as border points. A border point is the point which is density-connected to a border point in the same cluster. The steps of DBSCAN algorithm are shown in Table III.

Table III: Steps of DBSCAN algorithm.

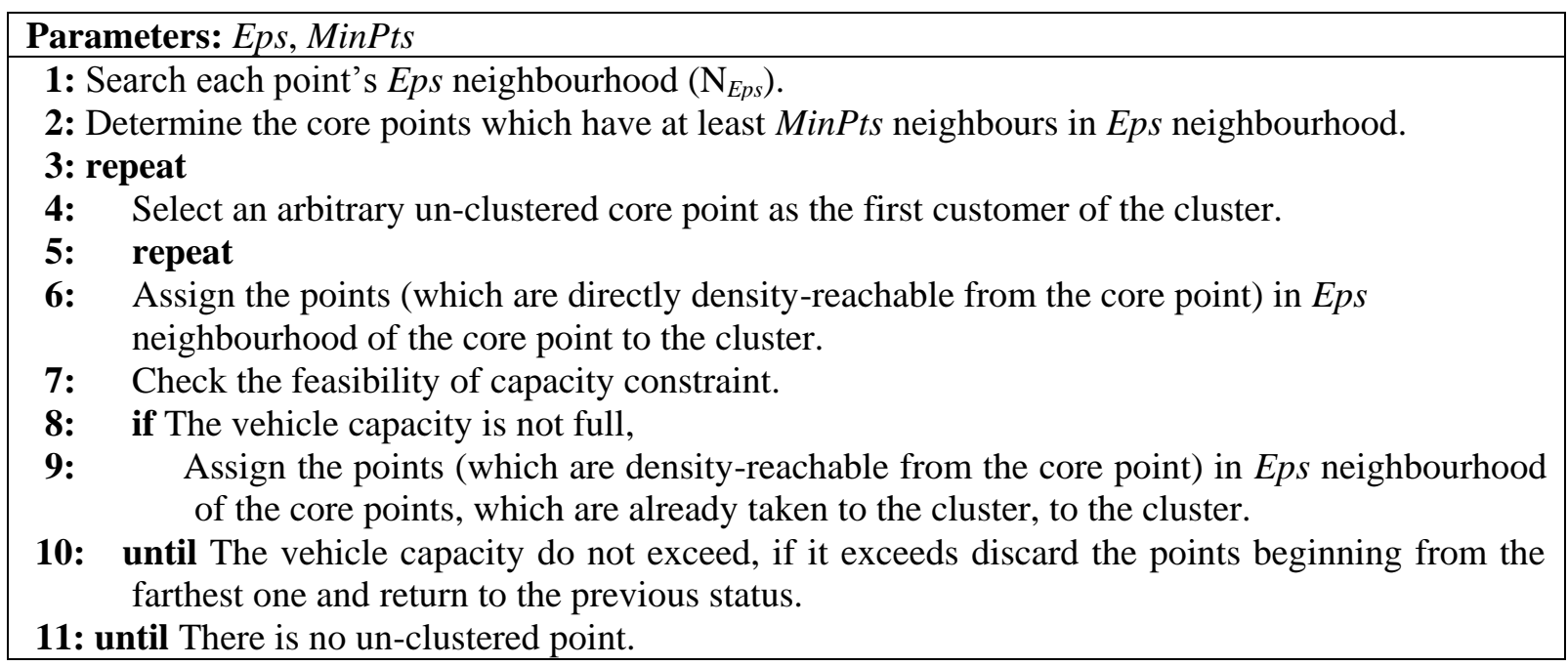

(4) Shared Nearest Neighbour

Shared Nearest Neighbour (SNN) clustering algorithm proposed by Ertoz et al. [17]. SNN algorithm is a density based clustering algorithm as DBSCAN algorithm. The main difference between them is the density concept. In SNN algorithm, the density is related not only the distance between points, but also the number of the nearest points that they share. The steps of SNN algorithm are shown in Table IV.

Table IV: Steps of SNN algorithm.

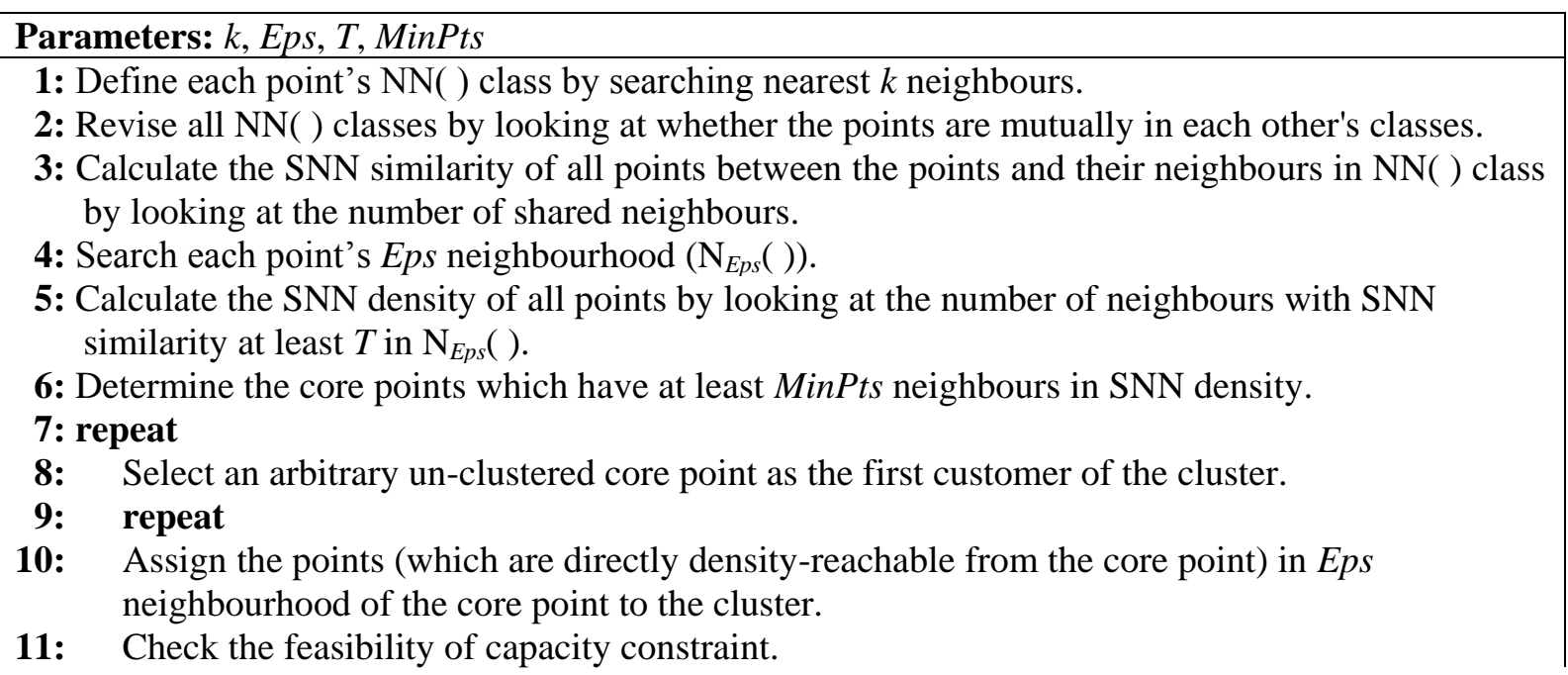


12: if The vehicle capacity is not full,

13: Assign the points (which are density-reachable from the core point) in Eps neighbourhood of the core points, which are already taken to the cluster, to the cluster.

14: until The vehicle capacity do not exceed.

15: until There is no un-clustered point.

\subsection{Routing phase}

The routing process occurs with the same rules for all algorithms. It begins with the inclusion of the depot in the clusters. At first, the depot is added and then the customer who is nearest to the depot in one cluster is assigned to the route. The distances between the non-routed customers and the last customer assigned to the route are computed. The closest one that provides the time constraint is assigned to the route. Thus, the routes are built for the customers at each cluster. If any non-routed customer exists, those who do not violate the limitations are assigned to a route. In the lack of a feasible location, a new route is formed. The practicable solutions are achieved via these improvements.

\subsection{Random-based initial algorithm}

For the observation of the effectiveness of the clustering algorithms at the generation of the initial population, a random-based algorithm is used for the comparison. Taking the time window constraint into account, the steps of the Random-based algorithm are indicated in Table V. The algorithm constructs the routes while selecting the customers.

Table V: Steps of Random-based algorithm.

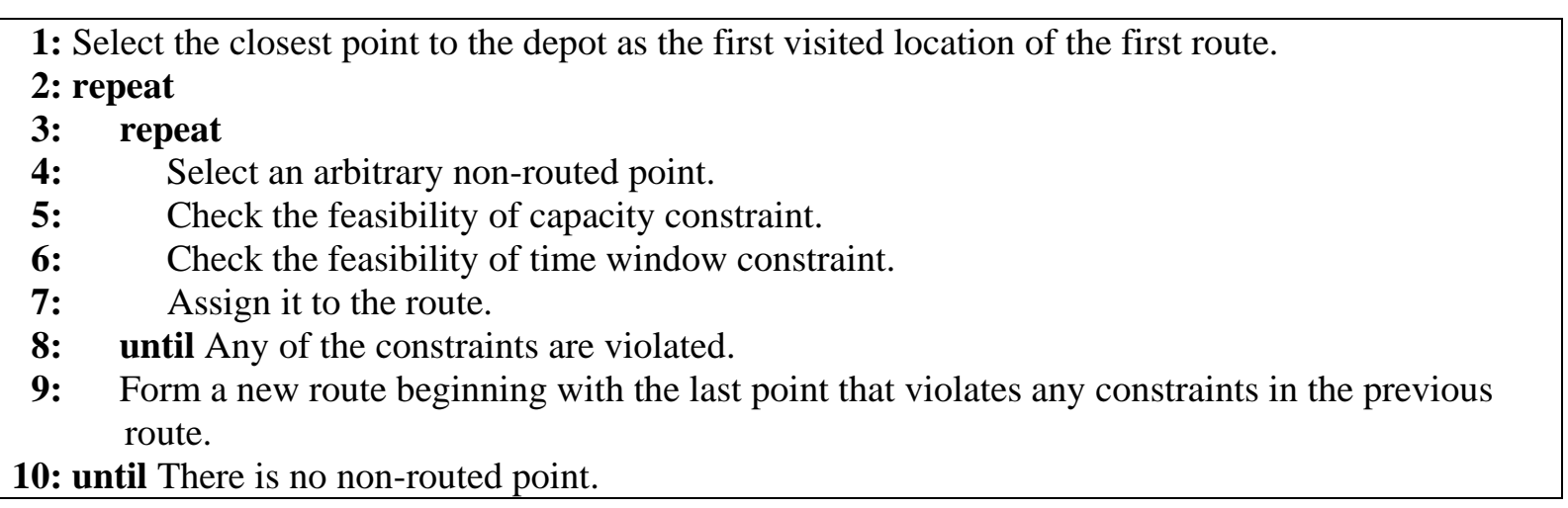

\subsection{Optimizing phase}

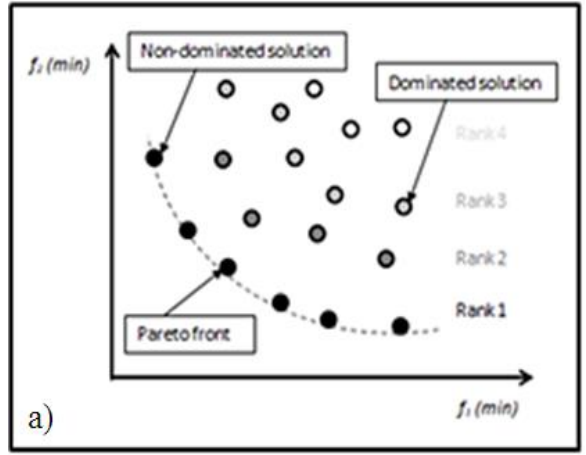

igure 1: a) Pareto front and rank

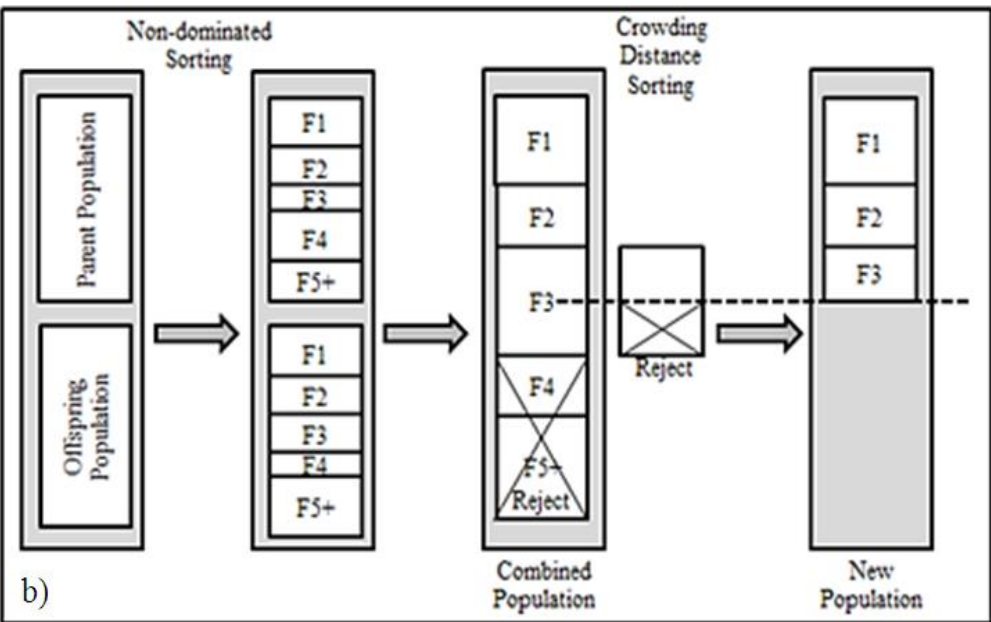

Population

Figure 1: a) Pareto front and ranking scheme, b) Elitist selection procedure of NSGA-II. 
After generating the initial population, GA is used for the optimization. NSGA-II (Nondominated Sorting Genetic Algorithm II) which is one of the multi objective optimization techniques is used in the evaluation, ranking and selection of the individuals at GA steps. DEAP Library is employed to perform NSGA-II $[18,19]$. The steps of the proposed GA are shown in Table VI.

Table VI: Steps of Genetic algorithm.

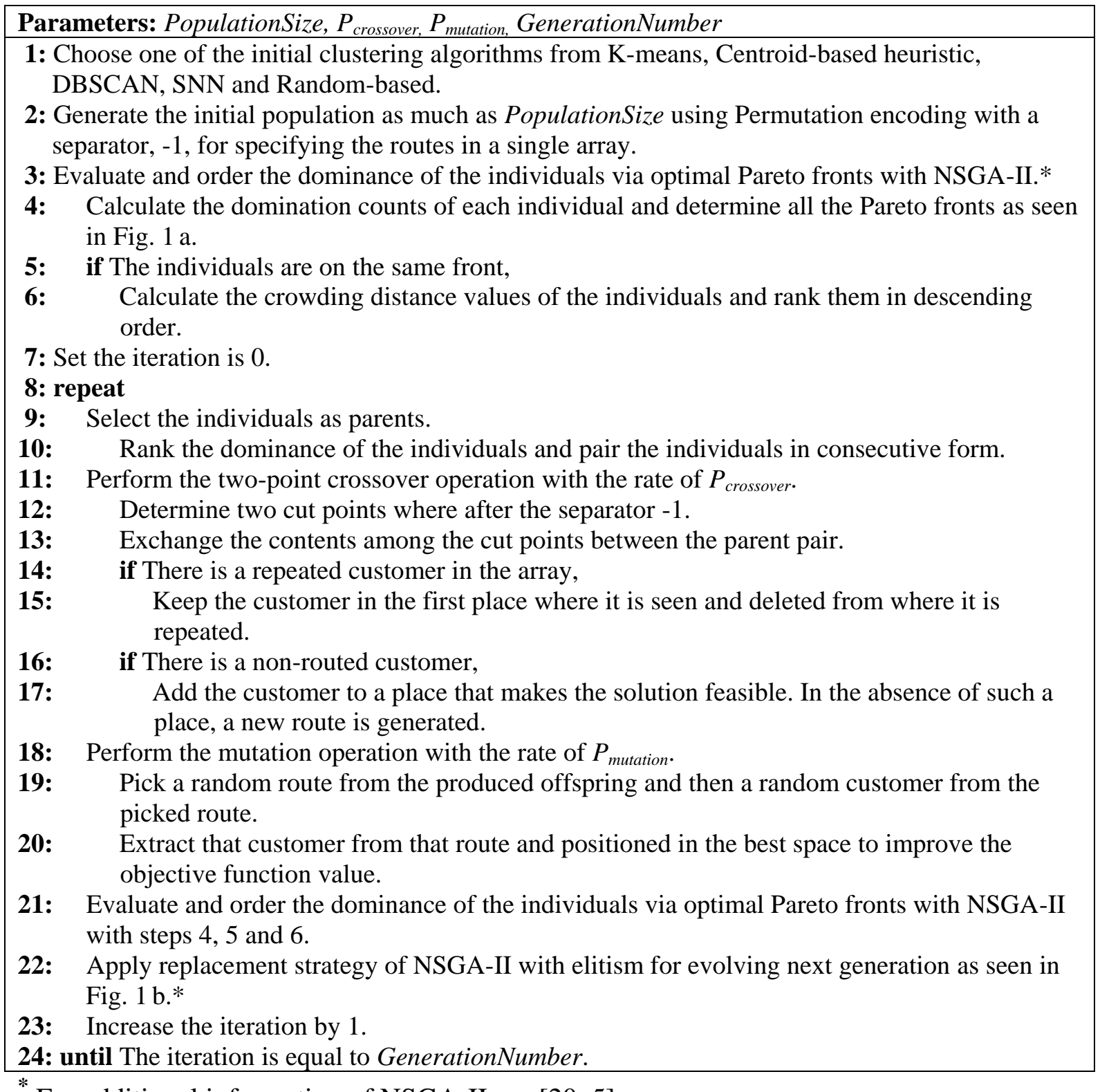

For additional information of NSGA-II see [20,5].

\section{NUMERICAL RESULTS}

The generated algorithms that utilize different clustering algorithms or a random-based algorithm are tested on C1, C2, R1 and R2 classes of Solomon's benchmark problems [21]. All problem instances have one depot, 100 customers and a fleet of homogeneous vehicles. Geographic positions of the customers and the depots are given by (x,y) coordinates. The route length between them is computed by Euclidean distance and the obtained value is in the unit of distance. It is assumed that the travelling of 1 unit distance takes 1 unit time. Customer demand quantities and service durations are available in the data set. The earliest and latest 
arrival times (i.e., time windows) of each customer are given. The problem classes differ according to the geographical data features and scheduling horizon type. In $\mathrm{C}$ category classes, the customers are clustered. In $\mathrm{R}$ category classes, the customers are distributed randomly and uniform. The problems in $\mathrm{C} 1$ and $\mathrm{R} 1$ classes have a short scheduling horizon; i.e., the time window of the depot is narrow; and low vehicle capacity. On the contrary, the problems in $\mathrm{C} 2$ and $\mathrm{R} 2$ classes have a long scheduling horizon; i.e., the time window of the depot is wide; and high vehicle capacity. The problem instances are available at [22].

The algorithms are coded in the Python 3.6. For testing the implementation, a computer with i5 processor technology, $3.00 \mathrm{GHz}$ processor speed and 8 GB RAM capacity is used.

The parameter $K$ which specifies the number of clusters in the K-means algorithm is calculated by adding 1 to the ratio of the total demand to the vehicle capacity. While forming the clusters by using K-means and Centroid-based clustering algorithms maximum iteration number is set to 10. The parameters of the DBSCAN algorithm are predetermined to be $E p s=12$ and MinPts $=4$. The parameters of the SNN algorithm are predetermined to be $k=40$, $E p s=12, T=1$ and MinPts $=5$. The parameter values used in the GAs are as follows: $P_{\text {crossover }}$ is always 0,7 and $P_{\text {mutation }}$ is 0,2 . Population size is 200 (at DBSCAN it is 300) and the number of generations is 200 (at SNN it is 300). The probability parameters are decided based on the values used in the literature. Other parameters used in GA and clustering algorithms are determined after preliminary runs of the problem.

For each problem set 5 independent tests are run. Algorithms gave the final solution populations as a result. Table VII is formed using the best distance values and Table VIII is formed using the best waiting time values on the Pareto optimal fronts of the final populations. These alternate Pareto solutions are comparable and decision maker can decide which solution is more preferable based on the preferences. The results are compared between the generated algorithms.

Table VII shows that K-means algorithm has attained better performance than other algorithms for 14 results which are shown with bold values according to the minimum travelled distance and minimum required total vehicle numbers. The following better performance belongs to CBased algorithm with 9 best results. Even so, K-means and CBased algorithms are equally competitive according to the difference between results, especially in the short scheduling horizon problems. The results reveal that DBSCAN and SNN (i.e., with density based clustering algorithms) are not as efficient as the algorithms with partitioning clustering algorithms; they show poor performances up to $7 \%$ according to the average total distance values of the classes. Random based algorithm is generally not as good as the other algorithms in particular in the $\mathrm{C} 1$ and $\mathrm{C} 2$ class problems. It shows poor performance up to $30 \%$ according to the average total distance values of the classes. It also gives up to $65 \%$ worse result for problem basis than K-means algorithm, in C208. However, RN algorithm results reveal that the algorithm is not as poor on random distributed customers as that of clustered customers. It reaches 4 best results in R 1 and 3 best results in R 2 class problems according to the total distance values, but it always requires more vehicles in total of the classes.

From Table VIII it is seen that the RN algorithm reached 33 best results out of 40 instance results in minimizing waiting time values ( 29 of them have 0 waiting time). It reveals better results in R1 and R2 class problems. However, it has not compensated for the travelled distance values and the required total vehicle number. According to the total of the travelled distance values, K-means algorithm achieves the best results in particular in the $\mathrm{C} 1$ and $\mathrm{C} 2$ class problems. Also, K-means algorithm reached 29 instance results with 0 waiting time. CBased algorithm performs approximately close to K-means algorithm on total travelled distance and waiting time values. DBSCAN and SNN algorithm results are not as efficient as 
the partitioning clustering algorithms. Moreover, required total vehicle numbers are minimum in K-means algorithm (with 332) and maximum in RN algorithm (with 343).

Figs. 2 and 3 display the class averages of the algorithms results according to the travelled distance and waiting time values. It enables the comparison of the data in Table VII and Table VIII. It is seen that a decrement in waiting time values causes an increment in travelled distance values. This fact can be observed in all problem instances and at all algorithms. In multi-objective optimization, an improvement of one of the objectives may cause deterioration of the other. Only in R1 class, the success of reducing waiting time values is not as good as of other classes. In general, the minimum averages of the results yielded by $\mathrm{K}$-means algorithm. Particularly in $\mathrm{C} 1$ and $\mathrm{C} 2$ classes, K-means algorithm reveals better results than the other algorithms. In R1 and R2 classes, the averages of RN algorithm results on total distance and waiting time values might be seen the minimum, but for the instance problem basis it does not dominate the others and requires more vehicles.
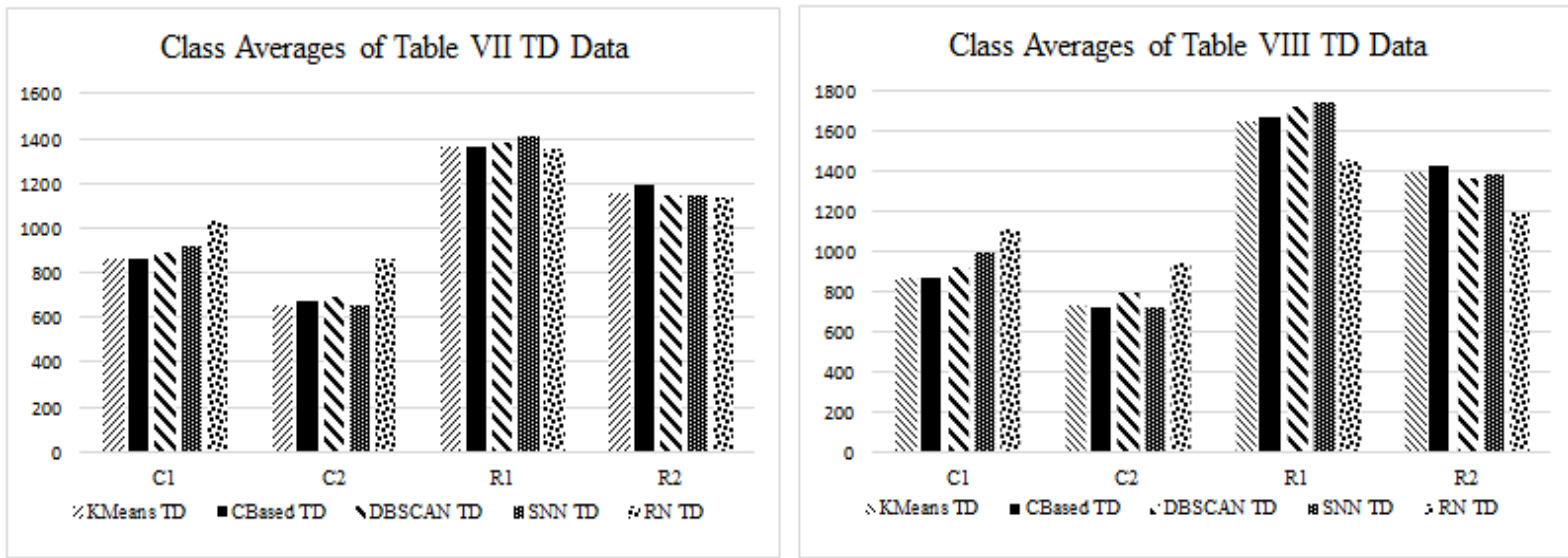

Figure 2: Comparison of the TD data in Table VII and Table VIII.
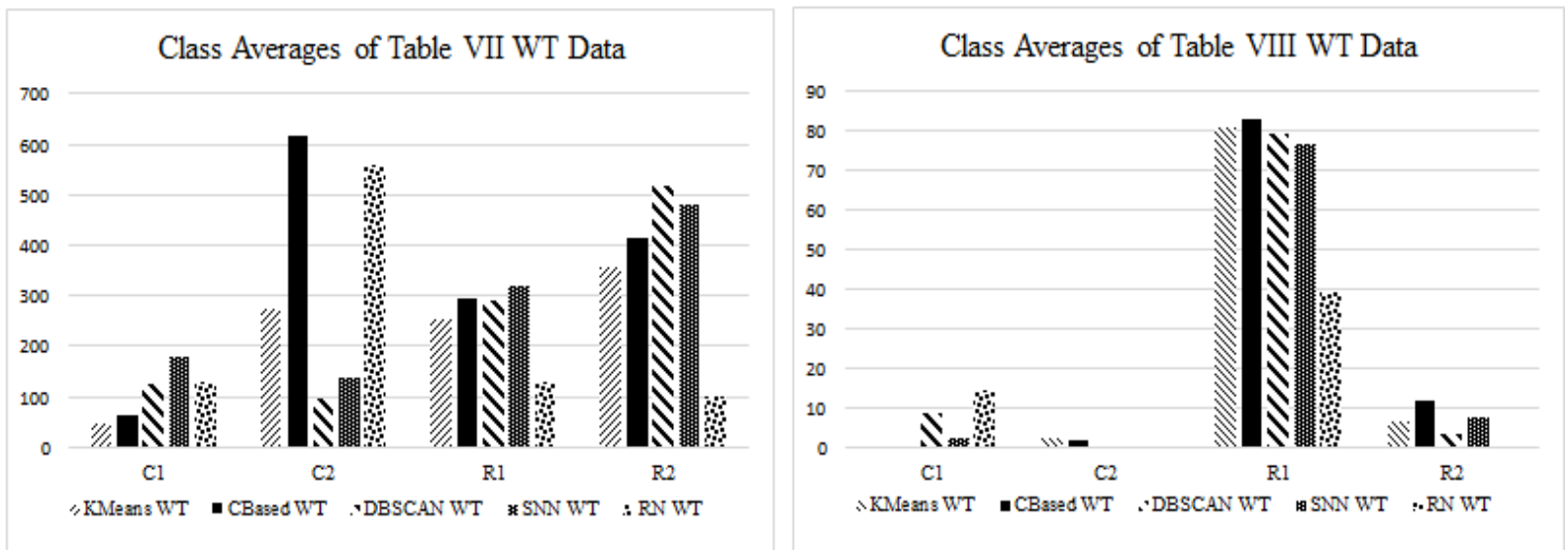

Figure 3: Comparison of the WT data on Table VII and Table VIII.

Fig. 4 indicates the average computational times (in minutes) of the algorithms for each class. K-means and CBased algorithms reach their results in less computational times. DBSCAN, SNN, and RN algorithms require almost twofold or threefold longer computational durations than K-means and CBased algorithms.

All the results of the tests show that K-means algorithm provided fast resolution, better total distance and waiting time values, effective competition with fewer vehicles. Nevertheless, it does not dominate over CBased algorithm. 
Gocken, Yaktubay: Comparison of Different Clustering Algorithms via Genetic Algorithm ...

Table VII: The solution results with the best distance values on the Pareto optimal fronts of the final populations obtained via the algorithms for each instances.

\begin{tabular}{|c|c|c|c|c|c|c|c|c|c|c|c|c|c|c|c|}
\hline \multirow{2}{*}{ Instance } & \multicolumn{3}{|c|}{ K-means } & \multicolumn{3}{|c|}{ CBased } & \multicolumn{3}{|c|}{ DBSCAN } & \multicolumn{3}{|c|}{ SNN } & \multicolumn{3}{|c|}{$\mathbf{R N}$} \\
\hline & $T D$ & $V N$ & $W T$ & $T D$ & $V N$ & $W T$ & $T D$ & $V N$ & $W T$ & $T D$ & $V N$ & $W T$ & $T D$ & $V N$ & $W T$ \\
\hline C101 & 828,94 & 10 & $\mathbf{0}$ & 828,94 & 10 & $\mathbf{0}$ & 934,71 & 11 & 592 & 830,36 & 10 & 22 & 968,98 & 11 & 380 \\
\hline C102 & 1971 & 11 & 221 & 911,03 & 11 & 449 & 970,89 & 11 & 390 & 1009,19 & 11 & 589 & 1170,97 & 12 & 128 \\
\hline C103 & 900,21 & 11 & 66 & 887,52 & 10 & 0 & 934,89 & 11 & 69 & 1005,16 & 11 & 453 & 1014,03 & 11 & 189 \\
\hline C104 & 883,85 & 10 & 128 & 888,23 & 10 & 128 & 928,71 & 10 & 68 & 1046,32 & 10 & 235 & 976,48 & 10 & 21 \\
\hline C105 & 828,94 & 10 & 0 & 828,94 & 10 & 0 & 833,24 & 10 & 0 & 838,07 & 10 & 0 & 1053,50 & 11 & 224 \\
\hline C106 & 828,94 & 10 & $\mathbf{0}$ & 846,16 & 10 & 0 & 850,46 & 10 & 0 & 846,16 & 10 & 0 & 1004,04 & 11 & 29 \\
\hline C107 & 828,94 & 10 & $\mathbf{0}$ & 828,94 & 10 & $\mathbf{0}$ & 880,84 & 10 & 0 & 838,07 & 10 & 0 & 1032,28 & 11 & 65 \\
\hline C108 & 871,58 & 10 & 0 & 871,58 & 10 & 0 & 853,52 & 10 & 0 & 891,43 & 10 & 44 & 996,45 & 11 & 71 \\
\hline C109 & 84,58 & 10 & 0 & 884,58 & 10 & 0 & 856,31 & 10 & 0 & 944,21 & 11 & 258 & 1124,75 & 11 & 58 \\
\hline C201 & 591,56 & 3 & $\mathbf{0}$ & 591,56 & 3 & 0 & 630,56 & 3 & 0 & 591,56 & 3 & 0 & 842,69 & 4 & 1044 \\
\hline C202 & 704,89 & 5 & 16 & 746,45 & 4 & 2559 & 746,87 & 3 & 34 & 678,59 & 4 & 9 & 839,06 & 4 & 839 \\
\hline C203 & 719,43 & 4 & 946 & 763,11 & 4 & 1676 & 708,68 & 4 & 179 & 741,52 & 4 & 467 & 975,07 & 4 & 422 \\
\hline C204 & 825,28 & 4 & 1245 & 822,34 & 4 & 715 & 782,12 & 4 & 459 & 765,88 & 4 & 618 & 830,00 & 4 & 308 \\
\hline C205 & 597,28 & 3 & 0 & 597,74 & 3 & 0 & 632,40 & 3 & 24 & & 3 & 0 & 782,72 & 4 & 428 \\
\hline C206 & 626,60 & 3 & 0 & 626,60 & 3 & 0 & 687,14 & 3 & 0 & 600,73 & 3 & 0 & 814,11 & 4 & 141 \\
\hline C207 & 599,86 & 3 & $\mathbf{0}$ & 606,37 & 3 & 0 & 670,47 & 3 & 62 & 614,57 & 3 & 0 & & 4 & 176 \\
\hline C208 & 599,28 & 3 & 0 & 598,65 & 3 & 0 & 662,01 & 4 & 0 & 626,47 & 3 & 0 & & 4 & 1134 \\
\hline R101 & 785,17 & 19 & 848 & 1816,32 & 21 & 1042 & 1819,82 & 20 & 830 & 1856,13 & 20 & 936 & 1861,53 & 21 & 460 \\
\hline R102 & 596,91 & 18 & 477 & 1690,09 & 18 & 571 & 1681,08 & 18 & 584 & 1724,28 & 18 & 530 & 1639,21 & 18 & 178 \\
\hline R103 & 01,75 & 15 & 318 & 1495,03 & 17 & 515 & 1455,93 & 15 & 394 & 1515,98 & 15 & 384 & 1420,18 & 16 & 207 \\
\hline R104 & 53,63 & 11 & 98 & 1160,10 & 11 & 139 & 1142,56 & 12 & 228 & 1186,48 & 12 & 375 & 1141,15 & 11 & $\mathbf{0}$ \\
\hline R105 & 79,18 & 15 & 260 & 1561,72 & 15 & 296 & 1533,83 & 15 & 261 & 1589,33 & 16 & 393 & 1587,80 & 16 & 226 \\
\hline R106 & 333,61 & 14 & 203 & 1453,81 & 14 & 207 & 1451,11 & 14 & 157 & 1511,68 & 14 & 146 & 1377,84 & 14 & 46 \\
\hline R107 & 261,82 & 12 & 120 & 1232,87 & 13 & 216 & 1264,28 & 13 & 123 & 1330,84 & 13 & 283 & 1257,75 & 13 & 105 \\
\hline R108 & 1050,29 & 11 & 102 & 1106,98 & 11 & 59 & 1135,08 & 11 & 172 & 1116,15 & 11 & 158 & 1073,72 & 11 & 0 \\
\hline R109 & 18,16 & 13 & 200 & 1324,35 & 13 & 140 & 1373,14 & 14 & 238 & 1349,14 & 13 & 187 & 1321,02 & 13 & 114 \\
\hline R110 & 229,01 & 12 & 105 & 1229,78 & 12 & 167 & 1305,84 & 13 & 138 & 1256,72 & 12 & 143 & 1281,80 & 13 & 54 \\
\hline R111 & 244,90 & 12 & 219 & 1222,61 & 12 & 84 & 1241,10 & 13 & 252 & 1332,42 & 13 & 196 & 1231,28 & 13 & 96 \\
\hline R112 & 75,18 & 11 & 113 & 1089,49 & 11 & 121 & 1134,05 & 11 & 108 & & 11 & 128 & 1103,03 & 11 & 58 \\
\hline R201 & 21,51 & 4 & 600 & 1537,10 & 4 & 834 & 1520,62 & 5 & 1168 & 1501,33 & 5 & 1135 & 1507,05 & $J$ & 447 \\
\hline R202 & 80,91 & 4 & 485 & 1404,33 & 4 & 400 & 1309,66 & 5 & 1156 & 1351,14 & 5 & 1064 & 1356,34 & 5 & 141 \\
\hline R203 & 32,20 & 4 & 410 & 1245,00 & 4 & 434 & 1172,81 & 4 & 603 & 1193,14 & 4 & 511 & 1208,84 & 4 & 0 \\
\hline R204 & 987,02 & 3 & 251 & 1020,69 & 4 & 614 & 1008,67 & 4 & 360 & 943,33 & 4 & 610 & 1014,00 & 4 & 80 \\
\hline R205 & 1248,12 & 3 & 331 & 1278,45 & 4 & 502 & 1212,99 & 3 & 110 & 1298,51 & 3 & 382 & 1195,72 & 3 & 67 \\
\hline R206 & 1184,13 & 4 & 351 & 1259,79 & 4 & 290 & 1134,15 & 3 & 325 & 1153,12 & 3 & 147 & 1107,45 & 4 & 230 \\
\hline R207 & 1073,49 & 4 & 499 & 1111,62 & 4 & 230 & 1007,53 & 3 & 284 & 1011,92 & 3 & 162 & 1034,94 & 3 & 25 \\
\hline R208 & & 3 & 268 & 870,01 & 3 & 187 & 873,56 & 3 & 105 & & 3 & 203 & 887,41 & 3 & 39 \\
\hline R209 & 1137,03 & 3 & 221 & 1173,20 & 3 & 188 & 1136,24 & 3 & 293 & 1171,62 & 3 & 302 & 1056,82 & 3 & 3 \\
\hline R210 & 1144,70 & 3 & 177 & 1207,22 & 4 & 604 & 1208,68 & 4 & 551 & 1199,28 & 4 & 521 & 1156,34 & 4 & 65 \\
\hline $\mathrm{R} 211$ & 958,21 & 3 & 323 & 973,81 & 3 & 280 & 990,49 & 4 & 766 & 957,97 & 3 & 236 & 981,87 & 3 & 7 \\
\hline
\end{tabular}

Remark: The bold values represent the best values among the 5 algorithms.

$T D$ - Total distance, $V N-$ Vehicle number, $W T$ - Waiting time of the vehicles. 
Gocken, Yaktubay: Comparison of Different Clustering Algorithms via Genetic Algorithm ...

Table VIII: The solution results with the best waiting time values on the Pareto optimal fronts of the final populations obtained via the algorithms for each instances.

\begin{tabular}{|c|c|c|c|c|c|c|c|c|c|c|c|c|c|c|c|}
\hline \multirow{2}{*}{ Instance } & \multicolumn{3}{|c|}{ K-means } & \multicolumn{3}{|c|}{ CBased } & \multicolumn{3}{|c|}{ DBSCAN } & \multicolumn{3}{|c|}{ SNN } & \multicolumn{3}{|c|}{$\mathbf{R N}$} \\
\hline & $T D$ & $V N$ & WT & $T D$ & $V N$ & WT & $T D$ & $V N$ & $W T$ & $T D$ & $V N$ & $W T$ & $T D$ & $V N$ & $W T$ \\
\hline C101 & 828,94 & 10 & $\mathbf{0}$ & 828,94 & 10 & $\mathbf{0}$ & 941,02 & 11 & 66 & 830,36 & 10 & 22 & 1027,93 & 11 & 49 \\
\hline C102 & 929,1 & 11 & $\mathbf{0}$ & 928,83 & 11 & $\mathbf{0}$ & 975,4 & 11 & $\mathbf{0}$ & 1013,26 & 11 & $\mathbf{0}$ & 1186,18 & 12 & 29 \\
\hline C103 & 919,65 & 11 & $\mathbf{0}$ & 887,52 & 10 & $\mathbf{0}$ & 996,85 & 11 & $\mathbf{0}$ & 1388,84 & 12 & $\mathbf{0}$ & 1137,16 & 11 & 8 \\
\hline C104 & 910,33 & 10 & $\mathbf{0}$ & 930,44 & 11 & $\mathbf{0}$ & 1130,23 & 11 & 12 & 1314,2 & 11 & $\mathbf{0}$ & 997,94 & 10 & 8 \\
\hline C105 & 828,94 & 10 & $\mathbf{0}$ & 828,94 & 10 & $\mathbf{0}$ & 833,24 & 10 & 0 & 838,07 & 10 & 0 & 1266,55 & 11 & 8 \\
\hline C106 & 828,94 & 10 & $\mathbf{0}$ & 846,16 & 10 & $\mathbf{0}$ & 850,46 & 10 & $\mathbf{0}$ & 846,16 & 10 & $\mathbf{0}$ & 1004,04 & 11 & 29 \\
\hline C107 & 828,94 & 10 & $\mathbf{0}$ & 828,94 & 10 & $\mathbf{0}$ & 880,84 & 10 & $\mathbf{0}$ & 838,07 & 10 & $\mathbf{0}$ & 1237,55 & 11 & $\mathbf{0}$ \\
\hline C108 & 871,58 & 10 & $\mathbf{0}$ & 871,58 & 10 & 0 & 853,52 & 10 & $\mathbf{0}$ & 915,97 & 10 & 0 & 1049,01 & 11 & 0 \\
\hline C109 & 884,58 & 10 & 0 & 884,58 & 10 & 0 & 856,31 & 10 & 0 & 995 & 10 & 0 & 1130 & 11 & 0 \\
\hline C201 & 591,56 & 3 & $\mathbf{0}$ & 591,56 & 3 & 0 & 630,56 & 3 & $\mathbf{0}$ & 591,56 & 3 & $\mathbf{0}$ & 1208,68 & 4 & 0 \\
\hline C202 & 806,13 & 5 & $\mathbf{0}$ & 835,5 & 4 & 11 & 1288,38 & 4 & $\mathbf{0}$ & 856,1 & 4 & $\mathbf{0}$ & 914,25 & 4 & 0 \\
\hline C203 & 1071,35 & 4 & 18 & 847,47 & 4 & 2 & 836,41 & 4 & $\mathbf{0}$ & 991,13 & 4 & 3 & 989,28 & 4 & 0 \\
\hline C204 & 1018,2 & 4 & 0 & 1084,68 & 4 & 0 & 999,16 & 4 & 0 & 933,83 & 4 & 0 & 863,27 & 4 & 0 \\
\hline C205 & 597,28 & 3 & 0 & 597,74 & 3 & 0 & 635,78 & 3 & 0 & 591,56 & 3 & 0 & 900,44 & 4 & 0 \\
\hline C206 & 626,6 & 3 & 0 & 626,6 & 3 & O & 687,14 & 3 & $\mathbf{0}$ & 600,73 & 3 & 0 & 842,21 & 4 & 0 \\
\hline C207 & 599,86 & 3 & $\mathbf{0}$ & 606,37 & 3 & O & 670,96 & 3 & $\mathbf{0}$ & 614,57 & 3 & $\mathbf{0}$ & 826,69 & 4 & $\mathbf{0}$ \\
\hline C208 & 599,28 & 3 & $\mathbf{0}$ & 598,65 & 3 & $\mathbf{0}$ & 662,01 & 4 & 0 & 626,47 & 3 & $\mathbf{0}$ & 997,98 & 4 & $\mathbf{0}$ \\
\hline R101 & 2156,14 & 20 & 539 & 1990,38 & 19 & 560 & 2326,89 & 21 & 542 & 2226,98 & 20 & 530 & 2037,22 & 20 & 253 \\
\hline R102 & 2188,5 & 19 & 204 & 2018,22 & 18 & 227 & 1972,03 & 18 & 228 & 2092,4 & 18 & 170 & 1953,31 & 21 & 94 \\
\hline R103 & 100,56 & 18 & 44 & 2232,16 & 18 & 39 & 2154,63 & 18 & 42 & 1859,57 & 15 & 54 & 1493,23 & 15 & 41 \\
\hline R104 & 361,58 & 11 & $\mathbf{0}$ & 1510,37 & 15 & $\mathbf{0}$ & 1737,28 & 14 & 0 & 2015,46 & 16 & $\mathbf{0}$ & 1141,15 & 11 & $\mathbf{0}$ \\
\hline R105 & 1881,27 & 16 & 125 & 1667,6 & 15 & 126 & 1720,93 & 15 & 96 & 1782,31 & 15 & 117 & 1726,65 & 16 & 78 \\
\hline R106 & 1713,14 & 15 & 11 & 1702,95 & 15 & 15 & 1699,77 & 15 & 13 & 1630,33 & 14 & 12 & 1495,67 & 15 & 0 \\
\hline R107 & 1557,42 & 14 & 0 & 1465,86 & 14 & 0 & 1867,66 & 17 & 0 & 1800,32 & 15 & 0 & 1285,27 & 13 & 0 \\
\hline R108 & 1097,78 & 11 & $\mathbf{0}$ & 1125,39 & 10 & $\mathbf{0}$ & 1210,05 & 11 & $\mathbf{0}$ & 1233,3 & 11 & $\mathbf{0}$ & 1073,72 & 11 & $\mathbf{0}$ \\
\hline R109 & 1457,17 & 13 & 38 & 1665,92 & 15 & 20 & 1630,11 & 14 & 21 & 1771,59 & 15 & 24 & 1498,43 & 14 & 5 \\
\hline R110 & 1614,71 & 14 & 4 & 1846,77 & 14 & 7 & 1725,97 & 14 & 5 & 1546,31 & 13 & 9 & 1345,17 & 13 & $\mathbf{0}$ \\
\hline R111 & 1547,4 & 14 & 4 & 1622,34 & 14 & 2 & 1423,34 & 13 & 7 & 1768,29 & 15 & 5 & 1318,03 & 13 & $\mathbf{0}$ \\
\hline R112 & 1204,87 & 11 & $\mathbf{0}$ & 1173,89 & 11 & $\mathbf{0}$ & 1214,46 & 11 & $\mathbf{0}$ & 1205,86 & 11 & $\mathbf{0}$ & 1119,81 & 11 & $\mathbf{0}$ \\
\hline R201 & 2034,7 & 4 & 47 & 2010,08 & 4 & 74 & 2089,09 & 4 & 29 & 2031,22 & 4 & 60 & 1928,24 & 4 & 0 \\
\hline R202 & 1748,68 & 4 & 23 & 1823,46 & 4 & 43 & 1903,71 & 4 & 9 & 1945,05 & 4 & 26 & 1408,18 & 5 & $\mathbf{0}$ \\
\hline R203 & 1631,6 & 4 & $\mathbf{0}$ & 1628,08 & 4 & 6 & 1379,5 & 3 & $\mathbf{0}$ & 1618,55 & 4 & 0 & 1208,84 & 4 & $\mathbf{0}$ \\
\hline R204 & 1238,79 & 3 & $\mathbf{0}$ & 1242,69 & 3 & 4 & 1184,61 & 3 & $\mathbf{0}$ & 1053,28 & 3 & $\mathbf{0}$ & 1027,16 & 3 & $\mathbf{0}$ \\
\hline R205 & 1467,16 & 3 & $\mathbf{0}$ & 1540,5 & 3 & $\mathbf{0}$ & 1415,84 & 3 & $\mathbf{0}$ & 1520,65 & 3 & $\mathbf{0}$ & 1250 & 3 & $\mathbf{0}$ \\
\hline R206 & 1281,59 & 3 & $\mathbf{0}$ & 1363,65 & 3 & $\mathbf{0}$ & 1263,4 & 3 & $\mathbf{0}$ & 1238,65 & 3 & $\mathbf{0}$ & 1145,06 & 4 & $\mathbf{0}$ \\
\hline R207 & 1159,62 & 3 & $\mathbf{0}$ & 1179,94 & 3 & $\mathbf{0}$ & 1064,87 & 3 & $\mathbf{0}$ & 1118,94 & 3 & $\mathbf{0}$ & 1046,36 & 3 & $\mathbf{0}$ \\
\hline R208 & 960,26 & 3 & $\mathbf{0}$ & 1009,82 & 3 & O & 953,48 & 3 & $\mathbf{0}$ & 928,7 & 3 & 0 & 888,85 & 3 & $\mathbf{0}$ \\
\hline R209 & 1369,04 & 3 & $\mathbf{0}$ & 1348,07 & 3 & 3 & 1288,25 & 3 & 1 & 1343,51 & 3 & 1 & 1082,36 & 3 & $\mathbf{0}$ \\
\hline R210 & 1336,17 & 3 & $\mathbf{0}$ & 1441,79 & 3 & 0 & 1357,18 & 3 & $\mathbf{0}$ & 1375,48 & 3 & $\mathbf{0}$ & 1232,42 & 4 & $\mathbf{0}$ \\
\hline R211 & 1120,49 & 3 & $\mathbf{0}$ & 1125,55 & 3 & 0 & 1133,69 & 3 & 0 & 1119,64 & 3 & $\mathbf{0}$ & 983,56 & 3 & 0 \\
\hline
\end{tabular}

Remark: The bold values represent the best values among the 5 algorithms.

$T D$ - Total distance, $V N-$ Vehicle number, $W T$ - Waiting time of the vehicles. 


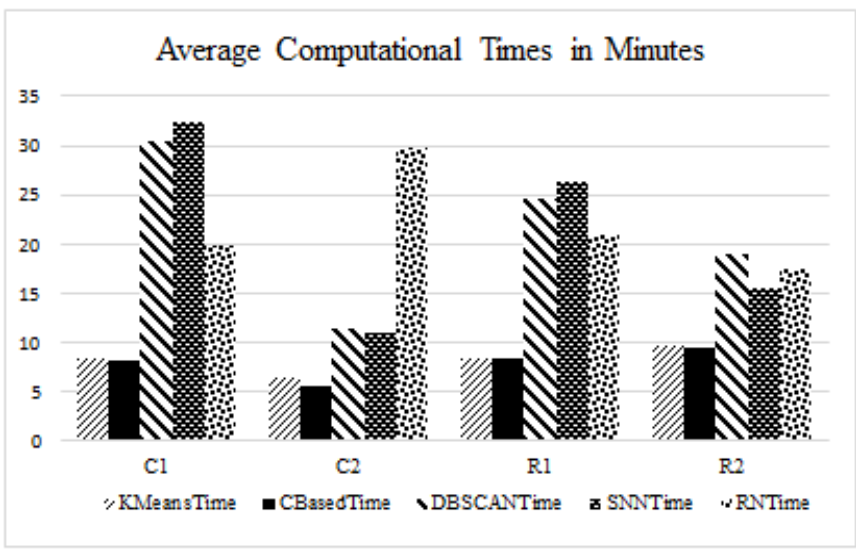

Figure 4: Average computational times of the algorithms for each class.

\section{CONCLUSION}

In this study, a multi objective GA approach for the VRPTW solution is proposed and the effect of using different clustering algorithms in the initial population generation step of GA is investigated. By using K-means, Centroid-based heuristic, DBSCAN and SNN clustering algorithms, the customers are divided into feasible clusters. Then feasible routes are constructed for each cluster. Lastly, the feasible route solutions are taken as the initial population and GA is utilized for the optimization. For the observation of the effectiveness of the clustering algorithms at the generation of the initial population, for comparison, a randombased algorithm is also used. The formed five algorithms; i.e. K-means, CBased, DBSCAN, SNN and RN; are tested on Solomon's VRPTW benchmark problems.

It can be concluded that the K-means algorithm showed better performance than other algorithms taking into consideration of the travelled distance, waiting time, vehicle number and computational time criteria. Nevertheless, it has not seen a significant difference between $\mathrm{K}$-means and CBased algorithms. They performed better than DBSCAN and SNN algorithms. Therefore, it can be interpreted that partitioning clustering algorithms are more appropriate for the VRPTW solution approaches than the density based clustering algorithms. Lastly, travelled distance results of the RN algorithm indicated that using clustering algorithms in the initial population generation step of GA have a positive effect on the results.

In the future studies, a parameter analysis can be made on the clustering algorithms for examining the influence on the algorithms performance. It may increase the efficiency of the DBSCAN and SNN algorithms. Furthermore, the effects of random selection of initial points for K-means algorithm can be analysed.

\section{REFERENCES}

[1] Baker, B. M.; Ayechew, M. A. (2003). A genetic algorithm for the vehicle routing problem, Computers \& Operations Research, Vol. 30, No. 5, 787-800, doi: $\underline{10.1016 / \mathrm{S} 0305-}$ $\underline{0548(02) 00051-5}$

[2] Karagul, K.; Gungor, I. (2014). A case study of heterogeneous fleet vehicle routing problem: Touristic distribution application in Alanya, An International Journal of Optimization and Control: Theories \& Applications, Vol. 4, No. 2, 67-76, doi:10.11121/ijocta.01.2014.00185

[3] Mousavi, M.; Yap, H. J.; Musa, S. N.; Dawal, S. Z. M. (2017). A fuzzy hybrid GA-PSO algorithm for multi-objective AGV scheduling in FMS, International Journal of Simulation Modelling, Vol. 16, No. 1, 58-71, doi:10.2507/IJSIMM16(1)5.368

[4] Zhao, P. X.; Luo, W. H.; Han, X. (2019). Time-dependent and bi-objective vehicle routing problem with time windows, Advances in Production Engineering \& Management, Vol. 14, No. 2, 201-212, doi:10.14743/apem2019.2.322 
[5] Gocken, T.; Yaktubay, M.; Kilic, F. (2017). Improvement of a genetic algorithm approach for the solution of vehicle routing problem with time windows, Proceedings of the 2017 International Artificial Intelligence and Data Processing Symposium, 8 pages, doi:10.1109/IDAP.2017.8090185

[6] Ghoseiri, K.; Ghannadpour, S. F. (2010). Multi-objective vehicle routing problem with time windows using goal programming and genetic algorithm, Applied Soft Computing, Vol. 10, No. 4, 1096-1107, doi:10.1016/j.asoc.2010.04.001

[7] Wu, D. Q.; Dong, M.; Li, H. Y.; Li, F. (2016). Vehicle routing problem with time windows using multi-objective co-evolutionary approach, International Journal of Simulation Modelling, Vol. 15, No. 4, 742-753, doi:10.2507/IJSIMM15(4)CO19

[8] Díaz-Parra, O.; Ruiz-Vanoye, J. A.; Zavala-Díaz, J. C. (2010). Population pre-selection operators used for generating a non-random initial population to solve vehicle routing problem with time windows, Scientific Research and Essays, Vol. 5, No. 22, 3529-3537

[9] Comert, S. E.; Yazgan, H. R.; Sertvuran, I.; Sengul, H. (2017). A new approach for solution of vehicle routing problem with hard time window: an application in a supermarket chain, Sädhanā, Vol. 42, No. 12, 2067-2080, doi:10.1007/s12046-017-0754-1

[10] Shin, K.; Han, S. (2011). A centroid-based heuristic algorithm for the capacitated vehicle routing problem, Computing and Informatics, Vol. 30, No. 4, 721-732

[11] López-Santana, E.; Rodríguez-Vásquez, W. C.; Méndez-Giraldo, G. (2018). A hybrid expert system, clustering and ant colony optimization approach for scheduling and routing problem in courier services, International Journal of Industrial Engineering Computations, Vol. 9, No. 3, 369-396, doi:10.5267/j.ijiec.2017.8.001

[12] Min, J. N.; Jin, C.; Lu, L. J. (2019). Maximum-minimum distance clustering method for splitdelivery vehicle-routing problem: Case studies and performance comparisons, Advances in Production Engineering \& Management, Vol. 14, No. 1, 125-135, doi:10.14743/apem2019.1.316

[13] Zhang, J. (2017). An efficient density-based clustering algorithm for the capacitated vehicle routing problem, Proceedings of the 2017 International Conference on Computer Network, Electronic and Automation, 465-469, doi:10.1109/ICCNEA.2017.96

[14] Erdogan, S.; Miller-Hooks, E. (2012). A green vehicle routing problem, Transportation Research Part E: Logistics and Transportation Review, Vol. 48, No. 1, 100-114, doi:10.1016/ j.tre.2011.08.001

[15] MacQueen, J. (1967). Some methods for classification and analysis of multivariate observations, Proceedings of the $5^{\text {th }}$ Berkeley Symposium on Mathematical Statistics and Probability, 281-297

[16] Ester, M.; Kriegel, H.-P.; Sander, J.; Xu, X. (1996). A density-based algorithm for discovering clusters in large spatial databases with noise, Proceedings of the Second International Conference on Knowledge Discovery and Data Mining (KDD'96), 226-231

[17] Ertoz, L.; Steinbach, M.; Kumar, V. (2003). Finding clusters of different sizes, shapes, and densities in noisy, high dimensional data, Proceedings of the 2003 SIAM International Conference on Data Mining, 47-58, doi:10.1137/1.9781611972733.5

[18] Deb, K. (2001). Multi-Objective Optimization Using Evolutionary Algorithms, John Wiley \& Sons, Chichester

[19] Fortin, F.-A.; De Rainville, F.-M.; Gardner, M.-A.; Parizeau, M.; Gagné, C. (2012). DEAP: evolutionary algorithms made easy, Journal of Machine Learning Research, Vol. 13, 2171-2175

[20] Deb, K.; Pratap, A.; Agarwal, S.; Meyarivan, T. (2002). A fast and elitist multiobjective genetic algorithm: NSGA-II, IEEE Transactions on Evolutionary Computation, Vol. 6, No. 2, 182-197, doi:10.1109/4235.996017

[21] Solomon, M. M. (1987). Algorithms for the vehicle routing and scheduling problems with time window constraints, Operations Research, Vol. 35, No. 2, 254-265, doi:10.1287/opre.35.2.254

[22] Solomon, M. M. (Northeastern University, Boston). VRPTW Benchmark Problems, from http://w.cba.neu.edu/ msolomon/problems.htm, accessed on 07-10-2019 